\title{
Conocimientos usuales de los pacientes diabéticos en los estudiantes de medicina del occidente de México"
}

Rafael Bustos Saldaña, Lizet Yuridia Florean Aguayo+ Gilberto López Hernández+ Evaristo Sánchez Novoa*+, Maria del Roció Palomera Palacios+,Alfonso Barajas Martínez, Rafael Bustos Mora +, Alejandro Bustos Mora+

*Hospital General de Zona con Medicina Familiar No. 9, del Instituto Mexicano del Seguro Social en Ciudad Guzmán, Jalisco, México. + Centro Universitario del Sur de la Universidad de Guadalajara, en Ciudad Guzmán, Jalisco, México

Objetivo: Identificar el conocimiento que presentan los estudiantes de Medicina acerca de los conceptos usuales de los pacientes diabéticos sobre su enfermedad.

Material y métodos: Estudio descriptivo en 275 estudiantes de Medicina del Centro Universitario del Sur de la Universidad de Guadalajara. Se seleccionó la muestra en forma aleatoria a los alumnos inscritos en el calendario 2006 A, para aplicarles el instrumento Diabetes Knowledge Questionnaire (24). Se utilizó el programa de estadística EPI INFO 2000, se tomó una significancia estadística $<0.05$.

Resultados: Edad en los estudiantes: $20.03 \pm 1.9$ años. El promedio total de contestaciones acertadas fue: estudiantes 17.56 \pm 3.24 , los alumnos de $7^{\circ}$ y $8^{\circ}$. ciclo presentaron $19.53 \pm 2.15$ y $19.75 \pm 1.92$ aciertos.

En cuanto a conocimientos básicos de la enfermedad solo el $\mathbf{7 5 . 8 2} \%$ contesto adecuadamente los reactivos,

Correspondencia:

Rafael Bustos Saldaña

Domicilio: Peñón No. 21

Colonia: Lomas Altas

Ciudad Guzmán, Jalisco, México

CP: 49064

Tel: 341-4134404

Email: rafaelb@cusur.udg.mx en el control glucémico $70.7 \%$ y en medidas de prevención y complicaciones $\mathbf{7 1 . 9 \%}$ de los estudiantes.

Conclusiones: El conocimiento de los estudiantes de Medicina en cuanto a los conceptos que deben de tener los pacientes diabéticos sobre su enfermedad se incrementa en forma significativa en cuanto aumenta los semestres de estudio, los primeros ciclos escolares tienen la tendencia a ser semejantes a la población de pacientes diabéticos de la región.

Palabras clave: Conocimiento en diabetes, estudiantes medicina.

Objective: To identify the knowledge that presents Medicine students about the usual concepts of the diabetic patients on their disease.

Material and Methods: Cross sectional study in 275 Medicine alumni of the University Center of the South of the University of Guadalajara. Sample was selected in randomized form in the students enrolled in calendar $2006 \mathrm{~A}$, in order to apply them instrument Diabetes Knowledge Questionnaire (24). The statistic program was used EPI INFO 2000, a statistical significance was taken $<0.05$.

Results: Students age: $20.03 \pm 1.9$ years. The total average of guessed right answers was: 17.56 \pm 3.24, students of $7^{\circ}$ and $8^{\circ}$. cycle presented $19.53 \pm 2.15$ 
and 19.75 \pm 1.92 right answers. As far as basic knowledge of the disease $75,82 \%$ answered the reagents suitably, in glycemic control $70.7 \%$ and prevention measures and complications $71.9 \%$. Conclusions: knowledge in Medicine students as far as the concepts that must have the diabetic patients on their disease increases in significant form as soon as it increases the semesters of study, the first scholastic cycles have the tendency to be similar to the population in regional diabetic patients.

Key Words: Diabetic Knowledge, Medicine Students

\section{INTRODUCCIÓN}

La Diabetes Mellitus es una pandemia que constituye un importante problema de Salud Pública en todo el mundo, tanto por el número de personas afectadas como por la implicación socioeconómica que presenta el control y tratamiento de la enfermedad y sus complicaciones ${ }^{1}$.

Se ha comprobado que la educación sobre diabetes reduce el riesgo de complicaciones del paciente, considerándose este aspecto el pilar fundamental del tratamiento ${ }^{2}$.

Los procesos educativos son la clave en las intervenciones preventivas en los pacientes, particularmente aquellos que han evolucionado de una relación emisor-receptor a una comunicación en la que el profesional de la salud comparte sus conocimientos y el receptor pasa de una actitud pasiva a otra activa y responsable ${ }^{3}$. Educar al paciente implica desarrollar en él los conocimientos, las destrezas, las motivaciones y los sentimientos que les permitan afrontar las exigencias terapéuticas de la diabetes con autonomía y responsabilidad, pero sin menoscabo del bienestar general, que es el objetivo principal en su manejo ${ }^{4}$, por lo que el personal de salud encargado de su control deberá de tener los conocimientos necesarios para brindarle la mejor atención posible, lo anterior conlleva a que se realice la promoción del aprendizaje en los pacientes diabéticos a través de procesos educativos los cuales generalmente están realizados por profesionales de la salud y estudiantes de Medicina en formación.

El estilo de vida de la población diabética puede ser modificado favorablemente, aplicando un sistema de prevención sencillo y económico en base a la edu- cación por parte de lo médicos y enfermeras de la atención de salud primaria ${ }^{5}$.

Actualmente los programas de estudio de todas las carreras de Medicina abarcan en gran medida a la diabetes mellitus como tema central, debido principalmente por su gran importancia en el impacto epidemiológico de todas las comunidades. Sin embargo, poco se toma en cuenta las experiencias y conocimientos básicos que pueda tener el alumno dentro de su formación.

En las instituciones educativas generalmente el alumno es motivado para el aprendizaje de conocimientos teóricos y prácticos (constituye en la mayor parte de los programas el verdadero valor de la enseñanza) científicos sobre diabetes mellitus, por lo que el estudiante se acostumbra a tener este tipo de saberes, descuidando en la mayoría de las ocasiones los elementos básicos que deben de saber los pacientes diabéticos para comprender su enfermedad y así poder incorporarlos a los conocimientos integrales dentro de su educación.

Se realizo una búsqueda sistemática de información acerca del conocimiento de los estudiantes de medicina sobre los conceptos usuales de los pacientes diabéticos y nos e encontraron referencias de sustento.

$\mathrm{Al}$ tener esta perspectiva, el objetivo de la investigación fue Identificar el conocimiento que presentan los estudiantes de Medicina del Centro Universitario del Sur de la Universidad de Guadalajara acerca de los conceptos que deben de saber usualmente los pacientes diabéticos sobre su enfermedad.

\section{MATERIAL Y METODOS}

Se realizó un estudio descriptivo en 275 estudiantes de la carrera de Medicina del Centro Universitario del Sur de la Universidad de Guadalajara, localizada en Ciudad Guzmán, Jalisco, al occidente de México.

El universo de estudio fue de 305 alumnos matriculados en los primeros 8 semestres del ciclo escolar 2006A. La muestra se realizó utilizando la fórmula de estudios descriptivos poblacionales de STAT CALC de EPI INFO, teniendo en cuenta una prueba piloto donde encontramos que el $12 \%$ de los entrevistados contestaron más del $80 \%$ del cuestionario en forma adecuadamente. Esta frecuencia se tomo como referente para el cálculo del tamaño de muestra, con un error del $10 \%$ de lo encontrado, un poder del $80 \%$ y una confiabilidad del $95 \%$, teniendo al final un total de 275 individuos.

La obtención de la muestra se hizo por un mues- 
treo aleatorio entre los estudiantes de la carrera de Medicina, que se encontraban vigentes en su matrícula. Los estudiantes seleccionados fueron localizados en sus respectivas aulas de clases durante horario habitual de las mismas.

El día de la realización de la investigación, previo consentimiento informado por parte de los individuos del estudio, se aplicó el instrumento Diabetes Knowledge Questionnaire $24(\mathrm{DKQ} 24)^{6}$, el cual ya había sido validado en población México - Americana que residían en Starr County, Texas. El DKQ 24 fue creado para evaluar las condiciones de conocimiento de los pacientes, antes de su aplicación fue adecuado al vocabulario de la población de la región sur del estadod e Jalisco, México. El instrumento es fácil y poco agobiante para los alumnos; su aplicación se hace máximo de 15 minutos. El instrumento utilizado se deriva de una versión original usada en "The Starr County Diabetes Education Study (19941998)", el cual contiene un total de 60 reactivos.

Las opciones ofrecidas de respuesta eran: si, no o no sé. Para el estudio se aceptaron aquellas que realmente estaban adecuadamente contestadas para cada item.

Los 24 reactivos que consta el DKQ 24 se agruparon en: a) Conocimientos básicos sobre la enfermedad (10 items), b) Control de la glucemia (7 items) y c) Prevención de complicaciones (7 items).

Análisis estadístico: La expresión de las características categóricas se realizó con frecuencia simple y porcentajes. Las variables discretas se expresaron con media \pm desviación estándar. Para la comparación de grupos se utilizó $\mathrm{X}^{2}$ y Análisis de Varianza. Se consideró una significancia estadística con una $\mathrm{p}<0.05$. Para el análisis de resultados se utilizó el programa de computación EPI INFO 2000.
Aspectos éticos: El trabajo fue autorizado por el Comité de Investigación del Centro Universitario del Sur de la Universidad de Guadalajara. Se observaron estrictamente los preceptos emitidos por La Convención de Helsinki y sus enmiendas posteriores para este tipo de estudios de investigación.

\section{RESULTADOS}

Las características generales de los individuos de estudio se encuentran expuestas en la tabla 1.

\section{RESPUESTA AL CUESTIONARIO DKQ 24}

CONOCIMIENTOS BÁSICOS DE LA ENFERME$\mathrm{DAD}$, en este rubro se consideraron los conocimientos acerca de: a) la ingesta de azúcar como causa de diabetes, b) falta de insulina como causa de diabetes, c) participación de los riñones en la etiología de la diabetes y d) su producción de insulina, e) herencia en los hijos de padres diabéticos, f) la posibilidad de cura de la enfermedad, g) clasificación de diabetes, h) ingesta de alimento y producción de insulina, i) importancia de escoger y preparar los alimentos, y j) diferencia entre los alimentos para diabéticos y personas normales.

La tabla 2 nos muestra el porcentaje de contestaciones adecuadas de acuerdo a cada uno de los ítems explorados en conocimientos básicos de diabetes por cada ciclo escolar

CONTROL GLUCÉMICO. Se comprendieron las siguientes secciones: a) hiperglucemia en el paciente sin ejercicio ni dieta, b) identificación de cifras de hiperglucemia, c) utilización de la glucosuria en el control del paciente diabético, d) ejercicio y producción de insulina, e) mayor importancia de los medicamen-

Tabla 1. Características generales de los estudiantes de Medicina

\begin{tabular}{lllll}
\hline Ciclo escolar & $\begin{array}{l}\text { Cantidad } \\
\text { de participantes }\end{array}$ & Edad * & Sexo femenino & Sexo masculino \\
\hline $1^{\circ}$ & 30 & $18.500 \pm 1.72$ & $16(53.33 \%)$ & $14(46.67 \%)$ \\
$2^{\circ}$ & 38 & $18.342 \pm 0.88$ & $20(52.63 \%)$ & $18(47.37 \%)$ \\
$3^{\circ}$ & 29 & $19.379 \pm 0.62$ & $17(58.62 \%)$ & $12(41.38 \%)$ \\
$4^{\circ}$ & 45 & $19.622 \pm 2.23$ & $24(53.33 \%)$ & $21(46.67 \%)$ \\
$5^{\circ}$ & 21 & $20.143 \pm 0.85$ & $12(57.14 \%)$ & $9(42.86 \%)$ \\
$6^{\circ}$ & 36 & $20.333 \pm 0.79$ & $19(52.77 \%)$ & $17(47.23 \%)$ \\
$7^{\circ}$ & 32 & $22.000 \pm 2.09$ & $15(46.87 \%)$ & $17(53.13 \% 9$ \\
$8^{\circ}$ & 44 & $21.659 \pm 0.91$ & $22(50 \%)$ & $22(50 \%)$ \\
totales & 275 & $20.03 \pm 1.91$ & $145(52.7 \%)$ & $130(47.3 \%)$ \\
\hline
\end{tabular}

$F=30.6, p<0.00001$ 
Tabla 2. Conocimientos Básicos sobre diabetes mellitus en los estudiantes de acuerdo a cada ciclo escolar

\begin{tabular}{|c|c|c|c|c|c|c|c|c|c|}
\hline \multirow[b]{2}{*}{ CONOCIMIENTOS } & \multicolumn{9}{|c|}{ CICLO ESCOLAR } \\
\hline & 10 & 20 & 30 & 40 & 50 & 60 & 70 & 80 & $\mathrm{p}$ \\
\hline $\begin{array}{l}\text { El comer azúcar no es } \\
\text { causa de diabetes }\end{array}$ & $8(26.6)$ & $18(52.63)$ & $15(51.73)$ & $30(33.33)$ & $18(85.71)$ & $27(75)$ & $19(59.37)$ & $35(79.54)$ & * \\
\hline $\begin{array}{l}\text { Diabetes es causada por } \\
\text { la falta de insulina }\end{array}$ & $22(73.33)$ & $25(65.79)$ & $24(82,75)$ & $33(73,33)$ & $12(57.14)$ & $19(52.78)$ & $15(46.87)$ & $16(36.36)$ & * \\
\hline $\begin{array}{l}\text { Diabetes no es causada porque } \\
\text { los riñones no expulsan la glucosa } \\
\text { del cuerpo }\end{array}$ & $16(53.33)$ & $31(81.58)$ & $19(65.52)$ & $40(88.89)$ & $21(100)$ & $36(100)$ & $31(96.88)$ & $42895.45)$ & * \\
\hline Los riñones no producen insulina & $19(63.33)$ & $30(78.95)$ & $29(100)$ & $45(100)$ & $21(100)$ & $36(100)$ & $32(100)$ & $43(97.75)$ & * \\
\hline $\begin{array}{l}\text { Siendo diabético puedo tener } \\
\text { hijos diabéticos }\end{array}$ & $26(86.67)$ & $36(94.74)$ & $28(96.56)$ & $44(97.78)$ & $21(100)$ & $36(100)$ & $32(100)$ & $42(95.45)$ & \\
\hline Diabetes mellitus no se cura & $27(90)$ & $35(92.1)$ & $28(96.56)$ & $44(97.78)$ & $21(100)$ & $35(97)$ & $32(100)$ & $41(93.18)$ & \\
\hline $\begin{array}{l}\text { Diabetes se divide } \\
\text { principalmente en tipo } 1 \text { y } 2\end{array}$ & $20(66.67)$ & $32(84.25)$ & $25(86.21)$ & $43(95.55)$ & $17(80.95)$ & $36(100)$ & $31(96.87)$ & $43(97.72)$ & * \\
\hline $\begin{array}{l}\text { Comer mucho estimula } \\
\text { a producir insulina }\end{array}$ & $15(50)$ & $26(68.429$ & $22(75.86)$ & $19(42.22)$ & $8(38.09)$ & $36(100)$ & $16(50)$ & $16(36.36)$ & * \\
\hline $\begin{array}{l}\text { Es igual de importante el escoger } \\
\text { los alimentos a ingerir que } \\
\text { la forma de prepararlos }\end{array}$ & $26(86.67)$ & $37(97.38)$ & $27(93.1)$ & $39(86.67)$ & $17(80.95)$ & $34(94.44)$ & $27(82.32)$ & $29(88.64)$ & \\
\hline $\begin{array}{l}\text { Los alimentos de los diabéticos } \\
\text { no deben de ser diferentes a los } \\
\text { de los demás }\end{array}$ & $4(13.33)$ & $10(26.32)$ & $6(20.69)$ & $18(40)$ & $8(38.09)$ & $20(55.55)$ & $16(50)$ & $17(38.64)$ & * \\
\hline
\end{tabular}

tos en comparación de la dieta, f) identificación de síntomas de hiperglucemia y de g) hipoglucemia.

La tabla 3 nos muestra el porcentaje de contestaciones adecuadas de acuerdo a cada uno de los ítems explorados en el control del paciente por cada ciclo escolar.

PREVENCION Y DETECCION DE COMPLICACIONES. En este grupo de conocimiento se incluyeron los ítems relacionados a: a) diabetes como causa de mala circulación, b) concepto de cicatrización en heridas de diabéticos, c) cuidado de corte de uñas en los pacientes diabéticos, d) uso de alcohol y yodo para aseo de heridas en el paciente, e) diabetes como causa de daño renal, f) diabetes como causa de pérdida de sensibilidad y g) uso de medias y calcetines en el diabético. La tabla 4 nos muestra el porcentaje de contestaciones adecuadas de acuerdo a cada uno de los ítems explorados en las prevención y detección de complicaciones por cada ciclo escolar.

\section{RESULTADOS GENERALES}

En forma general de todo el cuestionario, los alumnos contestaron un total de 4830 (73.18\%) de respuestas adecuadas. En cuanto a cada ciclo esco- lar los resultados fueron los siguientes: a) primero $403(55.97 \%)$, b) Segundo 602 (66.01), c) tercero 446 $(64.08 \%)$, d) Cuarto $807(74.72 \%)$, e) Quinto 381 $(75.59 \%)$, f) Sexto $697(76.42 \%)$, g) Séptimo 625 $(81.38 \%)$ y g) Octavo $869(82.29 \%)$ aciertos $\left(\mathrm{X}^{2}=\right.$ 272.81, $\mathrm{p}=0.000001$ ).

El promedio general de respuestas adecuadas en todo el cuestionario fue de $17.56 \pm 3.2$, por ciclo escolar los alumnos contestaron: a) Primer ciclo $13.43 \pm$ 3.04 , b) Segundo $15.84 \pm 3.48$, c) Tercero $15.38 \pm 2.47$, d) Cuarto $17.93 \pm 2.65$, e) Quinto $18.14 \pm 1.8$, f) Sexto $19.36 \pm 1.59$, g) Séptimo $19.53 \pm 2.15$ y h) Octavo 19.75 $\pm 11.92(\mathrm{~F}=28.33, \mathrm{p}<0.00001)$

\section{DISCUSIÓN}

De manera general, los proveedores de salud se quejan de la desidia y falta de motivación de la mayoría de los pacientes para cumplir con el tratamiento. En ocasiones, también se alude al desconocimiento de elementos esenciales del autocuidado diario por parte de los pacientes, $\mathrm{y}$ hasta de la falta de capacitación de los proveedores de salud para desarrollar su función de educación terapéutica ${ }^{4}$. 
Tabla 3. Conocimientos sobre el Control Glucémico de los pacientes diabéticos en los estudiantes de acuerdo a cada ciclo escolar.

\begin{tabular}{|c|c|c|c|c|c|c|c|c|c|}
\hline \multirow[b]{2}{*}{ CONOCIMIENTOS } & \multicolumn{9}{|c|}{ CICLO ESCOLAR } \\
\hline & 10 & 20 & 30 & 40 & 50 & 60 & 70 & 80 & $p$ \\
\hline $\begin{array}{l}\text { Aquel diabético sin dieta, ejercicio } \\
\text { y tratamiento médico le aumenta } \\
\text { la glucemia }\end{array}$ & $14(46.67)$ & $26868.42)$ & $21(72.41)$ & $35(77.78)$ & $17(80.95)$ & $31(86.11)$ & $26(81.25)$ & $33(75)$ & * \\
\hline $\begin{array}{l}\text { Identificación de nivel alto de } \\
\text { glucemia en valores de } 210 \mathrm{mg} / \mathrm{dl}\end{array}$ & $12(40)$ & $25(65.79)$ & $21(72.41)$ & $41(91.11)$ & $19(90.48)$ & $33(91.67)$ & 28 (87.5) & $42(95.45)$ & * \\
\hline $\begin{array}{l}\text { No es la mejor prueba para } \\
\text { valorar la glucemia la realización } \\
\text { de glucosurias }\end{array}$ & $11(36.67)$ & $23(60.53)$ & $18(62.07)$ & $33(73.33)$ & 17 (80.95) & $31(86.11)$ & ) 31 (96.87) & $43897.73)$ & * \\
\hline $\begin{array}{l}\text { El ejercicio no produce mayor } \\
\text { necesidad de insulina o } \\
\text { medicamentos }\end{array}$ & $20(66.67)$ & $32(84.21)$ & $18(62.07)$ & $34(72.55)$ & $18(85.71)$ & $30(83.33)$ & 27 (84.37) & $36(81.82)$ & \\
\hline $\begin{array}{l}\text { El medicamento no es mas } \\
\text { importante que la dieta y el ejercicio }\end{array}$ & $24(80)$ & $31(81.58)$ & $26(89.65)$ & $43(95.55)$ & $15(71.43)$ & $34(94.34)$ & 28 (87.5) & $40(90.91)$ & \\
\hline $\begin{array}{l}\text { No son datos de hiperglucemia } \\
\text { la sudoración y la sensación de frió }\end{array}$ & $6(20)$ & $5(13.6)$ & $5(17.24)$ & $12(26.6)$ & $10(47.629$ & $20(55.55)$ & $15(46.87)$ & $30(68.18)$ & * \\
\hline $\begin{array}{l}\text { No son datos de hipoglucemia } \\
\text { la presencia de polidipsia y polifagia }\end{array}$ & $6(20)$ & $16(42.1)$ & $13(44.83)$ & $25(55.55)$ & $16(76.19)$ & $30(83.33)$ & $27(84.37)$ & $38(86.36)$ & * \\
\hline
\end{tabular}

Si bien la educación es la piedra angular de la conducta clínica ante la diabetes, el personal de salud debe de estar lo suficientemente calificado y motivado en su cuidado para poder transmitir tanto los conocimientos como las habilidades y actitudes hacia la persona con la enfermedad ${ }^{8}$ y su entorno familiar y social ${ }^{9}$.

Una acción efectiva ante el problema de salud "Diabetes" exige un enfoque integrador de los aspectos de prevención, cuidados clínicos y educación del paciente. Sin embargo, aún resulta difícil encontrar un servicio de salud que ponga igual énfasis en los aspectos educativos que en los cuidados, a pesar de los resultados de las investigaciones realizadas en el campo de la diabetes mellitus demuestran, de manera fehaciente, que por ignorancia de los grupos de riesgo aumentan su prevalencia, por desconocimiento, falta de destrezas y de motivaciones para afrontar las exigencias terapéuticas ${ }^{10}$

Los médicos y el personal de salud (incluidos los alumnos de medicina) deben de enseñar a los pacientes los conocimientos, actitudes y prácticas necesarias para que estos lleven a cabo el mejor control de su enfermedad, si existe debilidad en el conocimiento que debe de manejar el paciente por parte del facultativo, muy probablemente se vera reflejado en una mala enseñanza de las indicaciones y por ende un ineficaz aprendizaje y un mal control por parte del paciente.
La educación del paciente diabético frecuentemente es realizada por las escuelas de medicina y enfermería de todas las instituciones educativas, ya sea a través de programas comunitarios o por iniciativa propia de los estudiantes.

La carrera de Medicina del Centro Universitario del Sur de la Universidad de Guadalajara cuenta en su curricula con 8 ciclos básicos de formación de los estudiantes, posterior a esto los alumnos pasan a realizar un año de rotación hospitalaria y realizan otro año de servicio social a la comunidad. Esta fue la causa por lo cual el estudio se realizó solamente en los estudiantes que se encontraban dentro de las instalaciones de la facultad de Medicina.

La actual curricula en la carrera de Medicina no muestra materias que traten de interpretar las actividades de los pacientes desde su punto de vista. Solamente se aborda la enfermedad desde el punto de vista científico en múltiples materias en forma integral, sin embargo, con nula visualización de aspectos usuales en la educación de los pacientes.

Durante el año 2005 y 2006 se realizaron varios estudios de investigación en la región con pacientes no diabéticos ${ }^{11,12,13}$ y diabéticos ${ }^{13-17}$, así como con sus familiares ${ }^{14-17,18}$ para conocer el conocimiento que presentaban sobre diabetes mellitus.

El presente estudio muestra que los conceptos básicos sobre Diabetes un nivel de conocimientos en los 
Tabla 4. Conocimientos sobre el Prevención y Detección de Complicaciones de los pacientes diabéticos en los estudiantes de acuerdo a cada ciclo escolar.

\begin{tabular}{|c|c|c|c|c|c|c|c|c|c|}
\hline \multirow[b]{2}{*}{ CONOCIMIENTOS } & \multicolumn{9}{|c|}{ CICLO ESCOLAR } \\
\hline & 10 & 20 & 30 & 40 & 50 & 60 & 70 & 80 & $\mathrm{p}$ \\
\hline $\begin{array}{l}\text { Diabetes mellitus causa problemas } \\
\text { de circulación sanguínea }\end{array}$ & $15(50)$ & $24(63.16)$ & $17(58.62)$ & $33(73.33)$ & $29(80.95)$ & $29(80.55)$ & 30 & $41(93.18)$ & * \\
\hline $\begin{array}{l}\text { Las heridas en los pacientes } \\
\text { diabéticos no cicatrizan más lento }\end{array}$ & $28(93.33)$ & $35(92.11)$ & $25(86.21)$ & $44(97.78)$ & $18(85.71)$ & $35(97.22)$ & $32(100)$ & $43(97.72)$ & \\
\hline $\begin{array}{l}\text { El paciente diabético debe de } \\
\text { tener más cuidado en el cortado } \\
\text { de sus uñas }\end{array}$ & $25(83.33)$ & $32(84.21)$ & $18(62.07)$ & $41(91.11)$ & $16(76.19)$ & $28(77.78)$ & 31 (96.87) & $44(100)$ & * \\
\hline $\begin{array}{l}\text { Las heridas de los diabéticos no } \\
\text { deben de lavarse a base de yodo } \\
\text { y alcohol }\end{array}$ & $2(6.67)$ & $2(5.269$ & $3(10.34)$ & $4(8.89)$ & $5(23.81)$ & $6(16.67)$ & $12(37.5)$ & $20(45.45)$ & * \\
\hline $\begin{array}{l}\text { Diabetes mellitus puede dañar } \\
\text { los riñones de los pacientes }\end{array}$ & $22(73.33)$ & $28(73.68)$ & $16(55.17)$ & $42(93.33)$ & $21(100)$ & $36(100)$ & $32(100)$ & $44(110)$ & * \\
\hline $\begin{array}{l}\text { Diabetes mellitus produce } \\
\text { alteraciones en la percepción } \\
\text { sensitiva }\end{array}$ & $18(60)$ & $26(68.42)$ & $10(34.48)$ & $36(80)$ & $15(71.43)$ & 33 (91.67) & $32(100)$ & $44(100)$ & * \\
\hline $\begin{array}{l}\text { Los pacientes diabéticos no deben } \\
\text { de utilizar medias elásticas } \\
\text { o calcetines apretados }\end{array}$ & $17(56.67)$ & $17(44.74)$ & $12(41.38)$ & $29(64.44)$ & $13(61.90)$ & $26(72.22)$ & $23(71.879$ & 37 (84.09) & * \\
\hline
\end{tabular}

alumnos de primer ciclo prácticamente igual al presentado por estudios realizados en pacientes diabéticos realizados en el sur de Jalisco, México ${ }^{11,14-17}$, a pesar de que los estudios realizados en los pacientes demuestran una escolaridad muy baja (primaria incompleta principalmente) por lo que el hallazgo de conocimiento sobre diabetes que exponen nuestros estudiantes universitarios de los primeros ciclos nos hacen reflexionar acerca de la poca educación en diabetes mellitus que pudiera tener las personas de las comunidades a las que pertenecen.

Uno de los conocimientos erróneos mas frecuentemente mencionados, es que el comer mucha azúcar o comidas dulces es causa de Diabetes, esto se observo en varios estudios realizados en comunidades de la región $\left(20.3 \%{ }^{12}, 19.66 \%^{13}, 20.83 \%^{14}, 21.6 \%{ }^{18}\right)$

Llama la atención los altos niveles de conocimiento en cuanto a la herencia de diabetes y la importancia de la selección de alimentos y la forma de prepararlos en el control del paciente. En este aspecto los alumnos contestaron mejor que los pacientes, probablemente la educación formal que han tenido los alumnos durante su proceso de aprendizaje ha tenido que ver en aspectos de dieta, por otra parte teniendo en cuenta la alta incidencia de diabetes mellitus en nuestra población ${ }^{19}$, era de esperarse que tendrían conocimiento sobre la herencia de esta enfermedad.
La relación de diabetes sin tratamiento y el factor hereditario en la Diabetes son aspectos con respuestas semejantes a lo observado en un estudio realizado al personal de salud ${ }^{3}$ y lo observado en la población de pacientes diabéticos y sus familiares de la región $\left(65 \%{ }^{14}, 57.77 \%{ }^{15}, 70.31 \%{ }^{18}\right)$, donde probablemente por experiencia de los alumnos por haber estado en contacto con familiares diabéticos pudieron estar influenciados para contestar acertadamente y no existió diferencia estadística significativa entre los distintos ciclos escolares.

La falta relativa de insulina juega un papel importante en la diabetes tipo 2, por lo que probablemente esta fue la causa para que los alumnos de los últimos ciclos no contestaran adecuadamente la respuesta acerca de la causa más frecuente de la diabetes mellitus, pensando en una deficiencia absoluta.

El resto de conocimientos básicos acerca de diabetes mellitus obtuvieron mejores calificaciones de acuerdo al incremento del ciclo escolar, muy probablemente secundario a que la curricula de la carrera de Medicina tiene contemplado varios cursos en los que se toma en cuenta esta patología

En el control glucémico de los pacientes los valores que presentaron los estudiantes de primer ciclo en general son muy precarios y diferentes a los observados por los pacientes de la región (tesis). Es 
importante recalcarla la falta de identificación adecuada de los síntomas de hiperglucemia e hipoglucemia, ya que la mayoría de los alumnos de Medicina, refieren que los temblores y sudoración son señales la primera; concepto erróneo y de gran importancia ya que son las complicaciones metabólicas agudas frecuentemente presentadas en los servicios de urgencias ${ }^{3}$.

Como se sabe la piedra angular en el manejo de los pacientes es las recomendaciones no farmacológicas y no la terapéutica médica en sí ${ }^{9}$. En este rubro es importante señalar que los estudiantes contestaron adecuadamente en su mayoría el valor que tienen los medicamentos con respecto a la dieta y el ejercicio.

Prácticamente en todos los conocimientos explorados acerca de la prevención y detección de complicaciones en los pacientes diabéticos, hubo una diferencia estadística significativa entre los primeros ciclos escolares y los finales. Es de llamar la atención que los alumnos tienen conocimiento no adecuado en todos los ciclos escolares acerca del aseo de las heridas en los pacientes a base de yodo y alcohol, ya que esta acción puede provocar irritación y edema de las mismas. Llama la atención que también los pacientes diabéticos de la región $\left(22 \%^{11}\right.$ $19.4 \%^{15}, 20.5 \%{ }^{14}$ ) contestaron en forma semejante a los estudiantes en este concepto, reforzando la importancia que tienen en los estudiantes los conocimientos adquiridos en la comunidad sobre su aprendizaje

El promedio de respuestas generales de los alumnos en el primer semestre fue muy similar a los observado en los pacientes diabéticos y sus familiares de la región $\left(12.8 \pm 3.5^{12}, 13.1 \pm 2.4^{13}, 14.2 \pm 4.2^{15}, 13.51 \pm 1.8^{16} \mathrm{y}\right.$ $\left.13.22 \pm 3.30^{18}\right)$. Este promedio se incrementa en forma significativa principalmente a partir del $3 \mathrm{er}$ y $4^{\circ}$ ciclo donde se inicia el abordaje de asignaturas clínicas en la curricula del programa de Medicina.

El propósito del trabajo no era la evaluación del incremento en conceptos científicos sobre diabetes mellitus en los estudiantes de medicina, sino el conocimiento que presentaban los alumnos de la carrera acerca de lo que deben de saber los pacientes diabéticos para que de esta manera potencialmente puedan comprenderlos mejor. Por lo que fue interesante el observar el incremento en conocimientos, indiscutiblemente secundarios a sus enseñanzas de la curricula, pero también, la falta de nociones en rubros que frecuentemente el paciente diabético pudiera cuestionarle al estudiante 0 durante su actividad profesional.

\section{CONCLUSIÓN}

El nivel de conocimientos que presentan los estudiantes de Medicina con respecto los conceptos que deben usualmente de tener los pacientes diabéticos sobre su enfermedad aumenta en forma proporcional de acuerdo a el incremento en sus estudios. En los primeros ciclos tiene la tendencia a ser semejante a los observado por los pacientes diabéticos de la región en otros estudios. Sin embargo, hasta los alumnos de $8^{\circ}$ semestre tienen deficiencias en el conocimiento en áreas básicas que los pacientes deberían de dominar.

\section{BIBLIOGRAFÍA}

1. Selli L, Papaleo LK, Meneghel SN, Torneros JZ.Educational techniques in diabetes treatment. Cad Saude Publica. 2005 SepOct;21(5):1366-72

2. Serra MP, Chichet A, Fernández M, Valdell L, Castrillón C, Giachero V et al. Prevalencia de diabetes en pacientes internados. Factores socioeconomicos-culturales: educación de la enfermedad y dificultades para el tratamiento. Revista Medica Uruguay 2003; 19:34-44

3. Aráuz A, Sánchez G, Padilla G, Fernández M, Rosello M, Guzmán S. Intervención Educativa comunitaria sobre la Diabetes en el ámbito de la atención primaria. Revista Panamericana Salud Publica 2001; 9: 142-148

4. Suárez PR, García GR, Aldana PD, Díaz DO, Grupo de intervención. Resultados de la educación en diabetes de proveedores de salud y pacientes. Su efecto sobre el control metabólico del paciente. Revista Cubana Endocrinología 2000;11:31-40

5. Quirantes HA, López LG, Curberto SV, Montaño LJ. La calidad de vida del paciente diabético. Rev Cubana Med Integr 2000; 16(1):50-6.

6. Garcia A, Villagomez E, Brown S, Kouzekanani K, Hanis C.The Starr county Diabetes Education study: Development of the Spanish-language Diabetes Knowledge questionnaire. Diabetes Care 2001;24: 16-21

7. Villagomez E: Health Beliefs, Knowledge, and Metabolic Control in Diabetic Mexican American Adults. MS thesis. Houston, TX, The University of Texas Health Science Center,1989

8. Ormen K, Svenkerud H, Klopstad A, Salminen L. Nurse education competence: A study of Norwegian nurse educators opinions of the importance and application of different nurse educator competence domains. Journal of Nursing Education 2002;Jul 41(7): 295-302.

9. Bustos-Saldaña R. Aprender para educar, una necesidad del médico de familia en la atención de los pacientes diabéticos. Rev Paceña Med Fam 2006;3(33):33-36

10. Suárez PR, García GR, Aldama PD, Díaz DO. Resultados de la educación en diabetes de proveedores de salud y pacientes. Su 
efecto sobre el control metabólico del paciente. Rev Cubana Endocrinol 2000; 11 (1):31-40.

11.Zamora CA. Conocimientos sobre diabetes mellitus en la población de la consulta del Centro de Salud Rural en Lagunillas Municipio de Atemajac de Brizuela, Jalisco, México. Tesis recepcional Médico Cirujano y Partero. Centro Universitario del Sur $2005 ; 45.48$

12. Guerrero GM. Conocimiento sobre diabetes en los adultos del programa Oportunidades de la localidad de Josefino de Allende, Jalisco, México. Tesis recepcional Médico Cirujano y Partero. Centro Universitario del Sur 2005; 30-45.

13. Ascencio TA. Conocimiento de los pacientes diabéticos tipo 2 y la población adulta sobre la enfermedades la población de Unión de Guadalupe, Jalisco, México. Tesis recepcional Médico Cirujano y Partero. Centro Universitario del Sur 2006: 32-47.

14. Corona PJ. Conocimientos sobre diabetes mellitus en pacientes diabéticos y sus familiares que acuden al Centro de Salud Urbano de Tecalitlán, Jalisco, México. Centro Universitario del Sur 2005: 45-50

15.Guzmán-Reyes C. Conocimientos sobre Diabetes Mellitus 2 en pacientes diabéticos y sus parejas que acuden a consulta externa en el centro de salud de el Cante Jalisco municipio de Autlan de Navarro. Tesis recepcional Médico Cirujano y Partero. Centro universitario del Sur 2005; 32-47

16. García-García M Conocimientos sobre Diabetes Mellitus en los pacientes diabéticos y sus familiares de Amacueca Jalisco. Tesis recepcional Médico Cirujano y Partero. Centro Universitario del Sur $2005 ; 29-48$

17. Mejía-Martínez E. Conocimientos en Diabetes Mellitus de los pacientes diabéticos y sus familiares que asisten a la consulta externa del Centro de salud de Pihuamo Jalisco de septiembre del 2004 a julio 2005. Tesis recepcional Médico Cirujano y Partero. Centro Universitario del Sur 2005; 31-48

18. Pizano-Moran R. Conocimientos sobre Diabetes en los familiares de los diabéticos tipo 2 de la población de Jiquilpan Jalisco. Tesis recepcional Médico Cirujano y Partero. Centro Universitario del Sur 2005: 29-48

19.Instituto Nacional de Estadística Geografía e Informática. México. XII Censo Nacional de Población y Vivienda 2000. México 2001. 